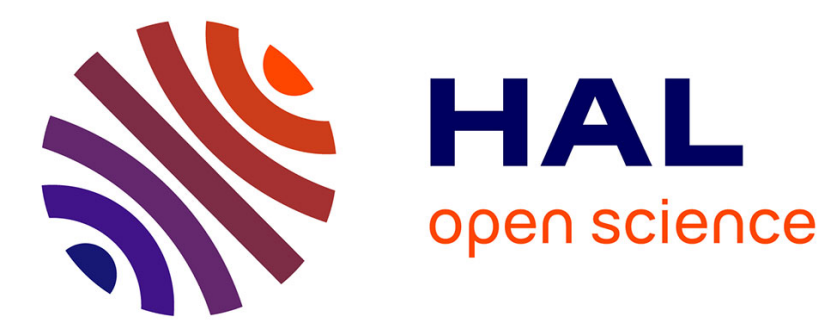

\title{
Physics and Modeling of Plasma Display Panels
}

\author{
J. Bøuf, C. Punset, A. Hirech, H. Doyeux
}

\section{To cite this version:}

J. Bœuf, C. Punset, A. Hirech, H. Doyeux. Physics and Modeling of Plasma Display Panels. Journal de Physique IV Proceedings, 1997, 07 (C4), pp.C4-3-C4-14. 10.1051/jp4:1997402 . jpa-00255558

\section{HAL Id: jpa-00255558 https://hal.science/jpa-00255558}

Submitted on 1 Jan 1997

HAL is a multi-disciplinary open access archive for the deposit and dissemination of scientific research documents, whether they are published or not. The documents may come from teaching and research institutions in France or abroad, or from public or private research centers.
L'archive ouverte pluridisciplinaire HAL, est destinée au dépôt et à la diffusion de documents scientifiques de niveau recherche, publiés ou non, émanant des établissements d'enseignement et de recherche français ou étrangers, des laboratoires publics ou privés. 


\title{
Physics and Modeling of Plasma Display Panels
}

\author{
J.P. Bøuf, C. Punset, A. Hirech and H. Doyeux*
}

Centre de Physique des Plasmas et Applications de Toulouse, Université Paul Sabatier, 118 route de Narbonne, 31062 Toulouse, France

* Thomson Tubes Électroniques, ZI Centr'Alp, Moirans, France

\begin{abstract}
We disciuss physical phenomena which take place in an AC Plasma Display Panel (PDP) cell for matrix and coplanar electrode geometries. The effect of the gas mixture and the electrode geometry on the breakdown voltage is first analyzed using a fluid discharge model. We then compare solutions from simple fluid models and more accurate hybrid models for a ID cell, and discuss the sensitivity of the models to the uncertainties on the charged particle transport coefficients. Finally we present typical results showing the space and time evolution of a discharge in AC matrix and coplanar cells.
\end{abstract}

\section{INTRODUCTION}

Plasma display panels (PDP's) are now the best candidate on the market in the competition for large size, wall hanging television displays [1], [2] . The feasibility of high quality $42 \mathrm{in}$. color plasma displays has been demonstrated by several companies, and mass production of these displays started in 1996. However, some of the characteristics of these displays can be improved, and basic research is needed to guide improvements in, for example, the luminous efficiency of PDP's [3] which is low, but acceptable, in today's devices. Modeling is an important part of this research effort since accurate and detailed models of the discharge and plasma formation in PDP cells are now available and have considerably improved our understanding of the physical mechanisms governing the light emission from a picture element [4], [5], [6], [7], [8], [9] .

In this paper we give a general presentation, based on numerical results from fluid and hybrid models, of the physical processes occurring in the plasma which forms in the PDP. In section 2 we describe the typical matrix and coplanar electrode geometries which are used in AC PDP's. The breakdown voltage in a PDP cell must be small in order to limit the cost of the electronic drivers. The breakdown voltage depends on the gas mixture and on the surface, which provides secondary electrons under ion bombardment. In a simple parallel plate geometry, the breakdown voltage is a function of the pd product - gas pressure times gap length (Paschen curve). This is no longer true in a coplanar geometry where the position of the third (address) electrode plays an important role. These questions are discussed in section 3. In section 4, results from fluid and hybrid models of a transient discharge occurring in a matrix PDP cell are compared. The aim of this comparison is to estimate the accuracy of simple fluid models based on the local field approximation (LFA). Although the LFA models are based on a very rough approximation, they are much more practical to use (much faster) than hybrid or particle models. This aspect is essential when the models are used for computer aided design. We show that in the conditions of a typical AC plasma display cell, the LFA model provides a good qualitative picture of the discharge, with a reasonable quantitative accuracy. We also show that the errors introduced by this approximation are not larger than those due to uncertainties in the data characterizing charged particle transport and secondary electron emission. In section 5 we describe the space and time evolution of a discharge in matrix and coplanar electrode geometry, on the basis of a 2D fluid model. 


\section{MATRIX AND COPLANAR AC PLASMA DISPLAYS}

In AC plasmas displays, the discharges occur between electrode stripes which are deposited on a glass plate and covered with a dielectric layer. In a matrix display, each discharge cell is at the intersection of a line electrode and a column electrode which are deposited on two parallel glass plates. The sets of line electrodes and column electrodes are orthogonal to each other. The matrix electrode geometry is represented in Figs. 1 and 2a. A discharge is fired by applying a large enough voltage between a line electrode $\mathrm{X}$, and a column electrode $\mathrm{Y}$.

In a coplanar display [10], [11], the discharges occur between electrodes which are parallel and are deposited on the same glass plate. A third electrode, the address electrode, is used to define the position of the discharge in the direction perpendicular to the parallel electrodes, and to trigger the discharge (see Fig. 2b). Dielectric barrier ribs are used to prevent electrical and optical interaction (cross-talk) between the cells. The barrier ribs also play an important role in the UV-visible photon conversion since a layer of phosphor is deposited on the rib walls. A picture element consists of three discharge cells with phosphors in the three fundamental colors.

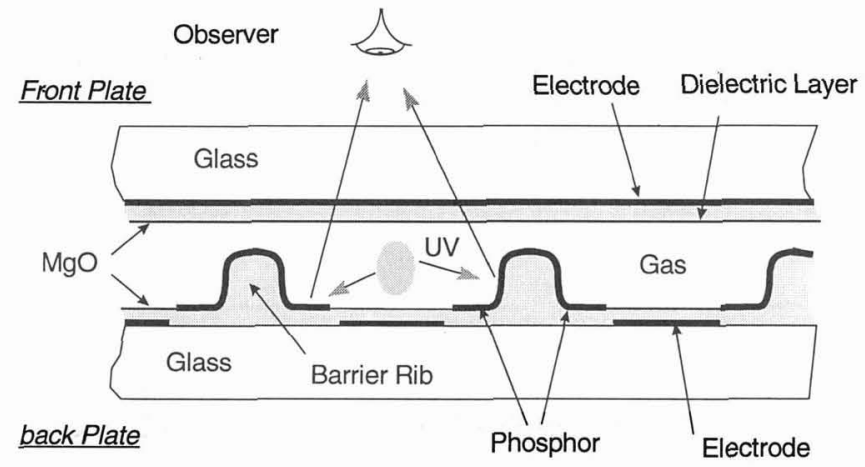

Figure 1: Schematic of an AC Matrix display (Thomson geometry)

The dielectric barrier ribs are parallel to the planes of Figs. $2 \mathrm{a}$ and $2 \mathrm{~b}$ and therefore do not appear on these figures (they are perpendicular to the plane of Fig. 1).

The dielectric layer above the electrodes is generally made of enamel and is typically $20-25 \mu \mathrm{m}$ thick. It is covered with a $\mathrm{MgO}$ layer (less than $1 \mu \mathrm{m}$ thick). The $\mathrm{MgO}$ layer is extremely important because 1) it protects the dielectric layer, and 2), its large secondary emission coefficient under neon or helium ion bombardment helps maintain a low breakdown voltage.

(a)

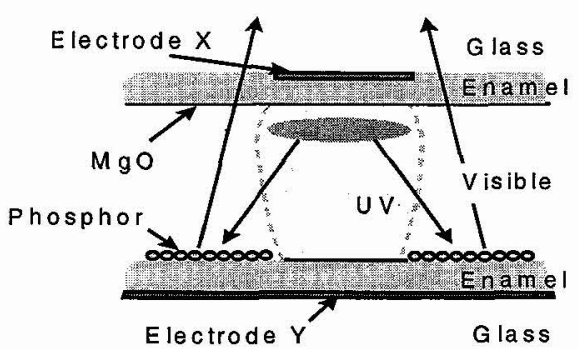

(b)
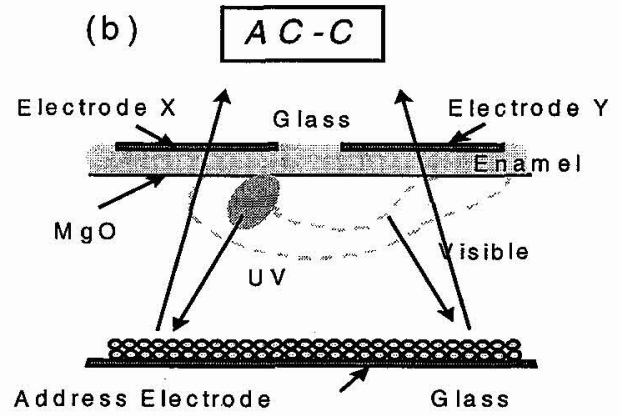

Figure 2: AC matrix (a), and coplanar (b) cells (cut in a plane parallel to the barrier ribs and perpendicular to the plane of Fig. 1)

Intense research efforts have been devoted to identifying materials which are resistant to sputtering and have a large secondary electron emission coefficient under ion bombardment. Magnesium oxide, 
$\mathrm{MgO}$, has been used for more than 30 years and is still the best known material for protecting the dielectric layer and providing secondary electrons. Uniform layers of $\mathrm{MgO}$ can be deposited on large areas by electron beam evaporation. An important advantage of $\mathrm{MgO}$ is that its secondary emission coefficient properties do not change significantly even after hundreds of nanometers are removed from the surface by ion sputtering. The life time of the display is limited by the sputtering of the MgO layer but can be larger than 10000 hours. The matrix and coplanar geometries are also called, respectively, double substrate and single substrate structures. We will not discuss here the advantages and drawbacks of each structure. However, let us mention that in the coplanar structure the electrodes on the front plate occupy a large area and must therefore be transparent (ITO, indium tin oxide). Due to the resistivity of ITO, a small width metal electrode (bus electrode) is generally deposited on the edge of each coplanar electrode to maintain a constant voltage along the electrodes.

\section{BREAKDOWN VOLTAGE}

In AC plasma displays, a square wave voltage, the sustaining voltage, is applied constantly between the $\mathrm{X}$ and $Y$ electrodes. The amplitude of the sustaining voltage is less than the breakdown voltage. In a matrix display, a discharge is turned on in a given cell by applying, between the electrodes of that cell, a voltage larger than the breakdown voltage (the writing voltage). The discharge forms and is quickly quenched because of the charging of the dielectric surfaces. At the next half cycle, when the sustaining voltage reverses, the field in the gas gap due to the charges deposited on the dielectric surfaces by the previous pulse now adds to the field due to the voltage across the electrodes and a new discharge is initiated. This discharge is in turn quenched by the charging of the dielectric surfaces and so on. If the charges on the dielectric layers at the end of a discharge pulse are $+Q$ and $-Q$, the next discharge must transfer $-2 Q$ and $+2 Q$ respectively on these surfaces, to maintain a steady state. The voltages of the writing and erasing pulses are such as to transfer exactly $+Q$ and $-Q$ to the dielectric surfaces. The sustaining voltage must be smaller than the breakdown voltage (otherwise all the cells would be constantly in an "ON" state). The sustaining voltage must also be large enough to maintain a written cell in the $O N$ state. The minimum and maximum values of the sustaining voltage define the margin of the display. It is important to operate in conditions where the margin is large because the operating value of the sustaining voltage must be within the margins of all the cells of the panel (which are not perfectly identical). As we have seen, the maximum value of the sustaining voltage is the breakdown voltage. The minimum value of the margin is related to (but always larger than) the minimum, normal glow discharge voltage. The calculations [4] show that the margin increases when the pd (pressure - gap length) product is increased. Therefore, the pd product must be large enough to have a large margin, but low enough to have a reasonably low breakdown voltage and sustain voltage.

In plasma displays, the sustaining and addressing pulses are generated by high-voltage transistors. High -voltage integrated drivers switch the addressing pulses to the appropriate electrodes. Choosing a gas mixture and electrode geometry leading to low operating voltages is therefore essential.

\subsection{Breakdown in Xe-Ne mixtures}

Although xenon is an efficient UV emitter, the breakdown voltage in pure xenon is impractically large and mixtures of xenon with neon or helium are generally used in plasma displays.

Adding neon or helium to xenon decreases the breakdown voltage for two reasons: 1) neon and helium ions are much more efficient than xenon at extracting secondary electrons from a $\mathrm{MgO}$ surface, and, 2) the total ionization coefficient in some xenon-neon mixtures for example, can be larger than the ionization coefficient in pure xenon and in pure neon (this is because the momentum cross-section for electron-neutral collisions is much larger in xenon than in neon so that in a mixture containing a large concentration of neon, the electrons can gain energy more easily and reach the excitation and ionization thresholds of xenon for lower values of the reduced electric field).

The above properties appear on Fig. 3 which shows the calculated Paschen curves for xenon-neon mixtures in a parallel plate geometry. The secondary electron emission coefficients in Fig. 3 are supposed 
to be independent of the reduced electric field (or ion energy). This figure shows that xenon-neon mixtures with xenon concentrations less than $10 \%$ are much more appropriate than pure xenon for plasma display cells. The secondary emission coefficients for neon and xenon ions on $\mathrm{MgO}$ are not well known although a value between 0.3 and 0.5 for neon ions seems reasonable [4]. The value of the secondary emission coefficients of xenon ions is not accurately known but it is undoubtedly much smaller than that of neon ions. We generally use a value of 0.05 in the calculations for xenon ions on $\mathrm{MgO}$, but this value is just a guess. Figure $3 \mathrm{~b}$ shows how the breakdown curve depends on the xenon secondary emission coefficient.

The breakdown curves of Fig. 3 have been obtained by from the self-sustaining condition in the gas mixture. If $\alpha_{\mathrm{Ne}}$ and $\alpha_{\mathrm{Xe}}$ are the partial ionization coefficients for neon and xenon, and if $\gamma_{\mathrm{Ne}}$ and $\gamma_{\mathrm{Xe}}$ are the secondary emission coefficients of $\mathrm{MgO}$ under neon and xenon ion bombardment this condition can be written:

$$
M=e^{\left[\alpha_{N e}+\alpha_{X e}\right] d}=1+\frac{\alpha_{N e}+\alpha_{X e}}{\alpha_{N e} \gamma_{N e}+\alpha_{X e} \gamma_{X e}}
$$
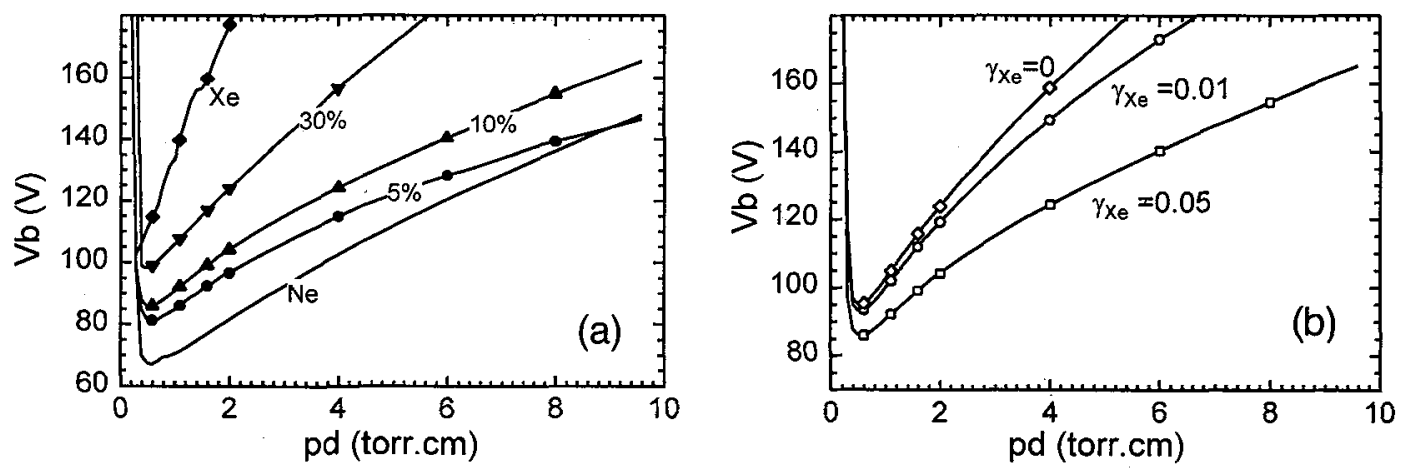

Figure 3: Breakdown curves in Xe-Ne mixtures for a parallel plate geometry (no dielectric layer above the electrodes). The secondary electron emission coefficient for neon ions on $\mathrm{MgO}$ is set to 0.5 in both figures. The secondary electron emission coefficient for xenon ions on $\mathrm{MgO}$ is set to $0.05 \mathrm{in}$ (a) and is varied in (b). The gas mixture is $\mathrm{Xe}-\mathrm{Ne}(10 \%-90 \%)$ in (b).

Note that Penning effect does not play a significant role in mixtures of neon with more than a few percent of xenon because most of the electron energy in these mixtures is spent into xenon excitation and ionization. Penning effect starts to become much more important when the concentration of xenon is less than $1 \%$. This is because for very low concentrations of xenon, most of the electron energy is deposited in excitation and ionization of neon. A lot of energy is stored in neon excited states and the ionization of a xenon atom by an excited neon atom becomes very probable. (Mixtures of neon with typically $0.1 \%$ argon have been used to lower the operating voltage in monochrome plasma display panels.)

\subsection{Breakdown in a coplanar geometry}

The breakdown condition above, for a parallel plate geometry, is a relation between the breakdown voltage and the pd product. This is because $\alpha / \mathrm{p}$ is a function of the reduced electric field $\mathrm{E} / \mathrm{p}=\mathrm{V} /(\mathrm{pd})$. This is exact for a parallel plate geometry (and if direct ionization by electron impact is the only ionization mechanism). In a matrix electrode geometry one can expect this to be true to first order. In a coplanar geometry the breakdown voltage no longer depends on the pd product alone, because the electric field distribution in the cell also depends on the position of the address electrode. The breakdown curves can still be plotted as a function of $p d$, but different curves are found for different $d$ and different $h$ where $d$ is the distance between the coplanar electrodes and $h$ is the distance between the dielectric surfaces.

The results displayed in Fig. 4 have been obtained with a 2D fluid model of the discharge cell shown in Fig. 4. Breakdown was said to have occured when the ion density in the cell reached $5.10^{12} \mathrm{~cm}^{-3}$ (for 
this value of the density, the plasma had not yet formed but the distortion of the geometric field due to the ion space charge was large enough to always lead to a further increase of the current).

We see on Fig. $4 a$ that the breakdown voltage increases considerably when the $d / h$ ratio is increased. This is because the low potential region between the bottom sustaining electrode and the address electrode becomes larger for larger $\mathrm{d} / \mathrm{h}$, and the secondary electrons are not efficiently drawn to the high field region between the two coplanar electrodes. A non-negligible fraction of the electrons emitted from the surface may be lost by diffusion for large $d / h$. We also see on Fig. 4a that the minimum of the breakdown curve is shifted toward larger pd when $d$ is increased. These tendencies have been observed in experiments [12], [13] but very few experimental data are available, and systematic comparisons are still to be done.
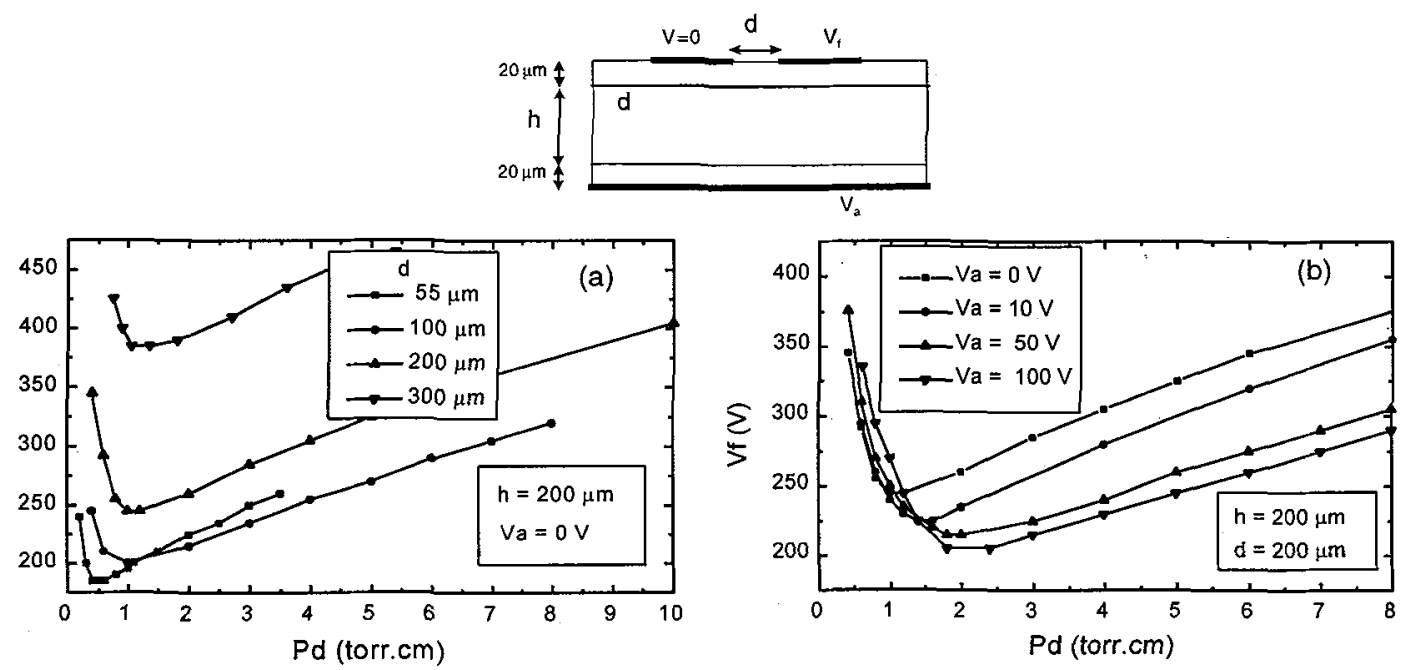

Figure 4: Breakdown curves in Xe-Ne mixtures in a coplanar structure ( 2 dimensional, Cartesian geometry). The secondary electron emission coefficient is set to zero on the dielectric surface above the addressing electrode. The thickness of both dielectric layers is set to $20 \mu \mathrm{m}$, and their relative permittivity is 12 . The secondary electron emission coefficients for neon and xenon ions on the $\mathrm{MgO}$ surface above the sustaining electrodes are supposed to be 0.5 and 0.05 , respectively.

The effect of the addressing voltage on the breakdown curve can be seen on Fig. $4 \mathrm{~b}$ where the gap length $\mathrm{d}$ and the distance between the plates $\mathrm{h}$ are kept constant. A non-zero voltage on the addressing electrode helps draw the secondary electrons emitted above the grounded sustaining electrode to the high field, multiplication region. The breakdown voltage can therefore be significantly decreased when $\mathrm{V}_{\mathrm{a}}$ is increased. We also observe a saturation effect on Fig. 4b: when the voltage of the addressing electrode is increased from $50 \mathrm{~V}$ to $100 \mathrm{~V}$, the effect on the breakdown voltage is relatively small.

The distances $\mathrm{d}$ and $\mathrm{h}$ characterizing the coplanar geometry must be chosen extremely carefully since 1) when $d / h$ is too large, the breakdown voltage becomes too large, and 2 ), when $d / h$ is too small, the value of $\mathrm{V}_{\mathrm{a}}$ does not affect strongly the discharge between the sustain electrodes and addressing becomes difficult. Note that $3 \mathrm{D}$ effects may also affect the breakdown curves in a real cell.

\section{PRINCIPLES AND ACCURACY OF THE MODELS}

The aim of the models is to provide a good qualitative understanding of the physical mechanisms occurring in a discharge cell and to help define the optimal cell geometry and operating conditions. It is clear that exact descriptions of such a complex system are not possible, and the main task of the modeler is to choose the necessary approximations in such a way that all the elements are on similar levels of accuracy. In this section we first try to estimate the accuracy of a simple fluid model by comparing its results with those from a more sophisticated (but much more time consuming) hybrid fluid - Monte Carlo model. The approximate description of the charged particle transport is the first source of error in the 
results. A second source of errors is related to the data which are used in the model (cross-sections, transport coefficients, secondary electron emission coefficients etc.). In the second part of this section we estimate the errors in the results which are due to uncertainties in the data and show that they are as large or larger as those due to the approximations in the transport equations implied by the LFA fluid model.

\subsection{Fluid and Hybrid models}

The models we are using to describe the discharge evolution in a plasma display cell have been previously described in Meunier et al. [4] , Boeuf and Pitchford [6], and Punset et al. [9] . Similar models have been developed by other groups [7], [14], [8]. They are based on solutions of the charged particle transport equations coupled with Poisson's equation for the electric field. They can be coupled to a model of the excited species kinetics in the xenon-neon mixture (see Meunier et al. [4] ) but the results show that, in typical conditions, the concentration of excited species in the discharge is relatively low and that second kind collisions (e.g. stepwise ionization) or Penning ionization do not play a significant role. The electric part of the model (charged particle transport and field) can therefore, to the first order, be decoupled from the excited species kinetics and the photon transport model.

The discharge is strongly collisional and fluid models seem more appropriate, under these conditions, than particle models. We choose to represent the charged particle momentum transport equation by the usual drift-diffusion approximation which is well suited to collisional situations. We also often replace the electron energy equation with the so-called local field approximation (LFA) which assumes that the electron energy losses exactly balance the electron energy gain, locally in space and time. This approximation is excellent in the case of a swarm of charged particles moving in a uniform or slightly non uniform field. It is, however, well known that it becomes less suitable when the relative variations of the electric field are not negligible over lengths on the order of the electron mean free path for energy exchange or during times on the order of the electron energy relaxation time. The cathode region of a DC or RF glow discharge is a typical example of where the LFA is not valid.

Since the discharge in an AC plasma display cell is quickly quenched because of the charging of the dielectric layers, the sheath length is not as small as it would be in DC conditions, and it is interesting to study quantitatively the errors which are induced by the local field approximation in that case. We therefore compared the results from two different discharge models. The first one is a fluid model based on the LFA and is similar to the models used in Meunier et al. [4] and Punset et al. [9].
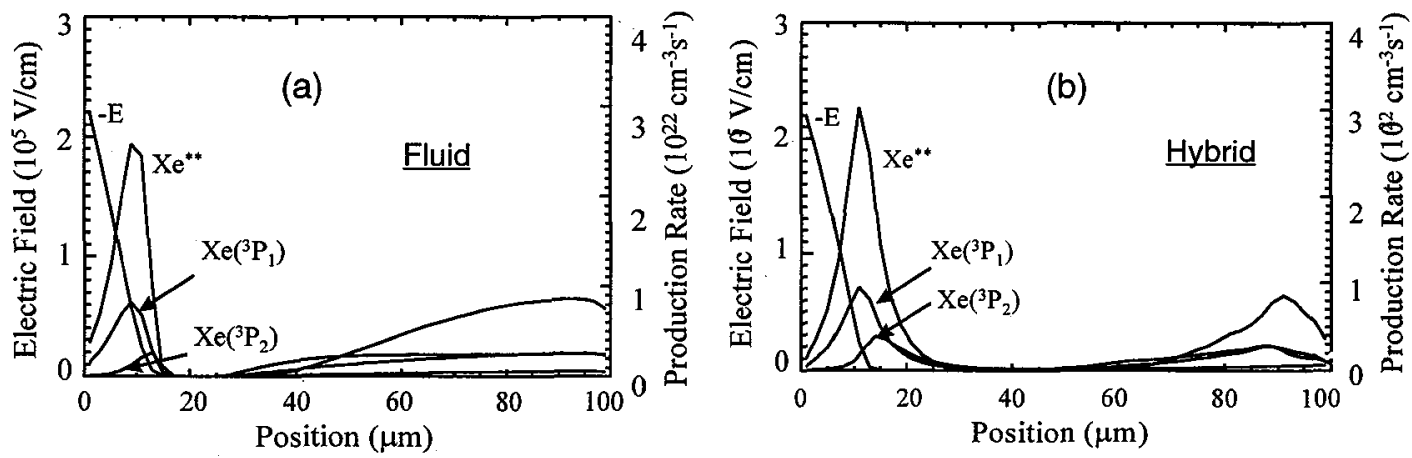

Figure 5: Spatial distributions of the excitation rates and electric field at the time of peak current from (a), the fluid, LFA model, and (b), from the hybrid fluid - Monte Carlo model (see text for the conditions).

The second model we have developed is a hybrid fluid - Monte Carlo model where the LFA is no longer used to calculate the ionization rate. The ionization rate in the hybrid model is obtained from.a Monte Carlo simulation of the electrons which are energetic enough to ionize and excite the xenon and neon atoms. In other words the tail of the electron distribution function is described with a particle, Monte Carlo simulation, while the transport of the bulk electrons is described with a simple, drift-diffusion, fluid 
model. Similar hybrid models have been described previously in J.P. Boeuf and L. Pitchford [15], and Fiala et al. [16]. The Monte Carlo simulation is run each time the electric field profile has changed by more than a given amount (a few percent). Each Monte Carlo simulation is performed assuming quasisteady state. This is possible because the relaxation time of the tail of the electron distribution function is fast compared to the characteristic time of variations of the electric field in the cell (this time is on the order of $1 \mathrm{~ns}$ in typical conditions while the electron collision time is on the order of $10^{-12} \mathrm{~s}$ and the energy exchange time for inelastic collisions is on the order of $10^{-10} \mathrm{~s}$ ).

We have compared the results of the fluid and hybrid model for a 1D (matrix) cell in the following conditions: Xe-Ne 10\%-90\% mixture, gas pressure 560 torr, $100 \mu \mathrm{m}$ gas gap, equivalent capacitance of the dielectric layers $230 \mathrm{pF} / \mathrm{cm}^{2}$, applied voltage between the electrodes $330 \mathrm{~V}$ and no memory charges on the dielectric surface. The ionization coefficients which have been used in the fluid LFA model in the results of Figs. 5, 6, and 8a have been obtained from Monte Carlo simulations in a uniform field. In the results of Figs. 7 and $8 \mathrm{~b}$, the ionization coefficients are those of Meunier et al. [4] which were derived from a multiterm Boltzmann equation. They are slightly different (up to $10 \%$ at high reduced field) from the ionization coefficients obtained with the Monte Carlo simulation in the hybrid model, under conditions of uniform electric field. This point will be discussed in a forthcoming paper.

Figure 5 shows a comparison between the predictions of the fluid and hybrid models at the instant of peak current. The spatial distributions of the electric field and xenon excitation rates at that time are represented. At this time, the sheath length is at its minimum and the electric field on the dielectric surface is maximum. These conditions are therefore the worst possible conditions for the local field approximation. We see that the field profiles predicted by the two models are very similar. The xenon excitation rates have similar profiles although, as expected, the hybrid model predicts a significant excitation in the zero field region, at the entrance of the negative glow plasma, while excitation on the cathode side occurs only in the sheath, in the fluid model.
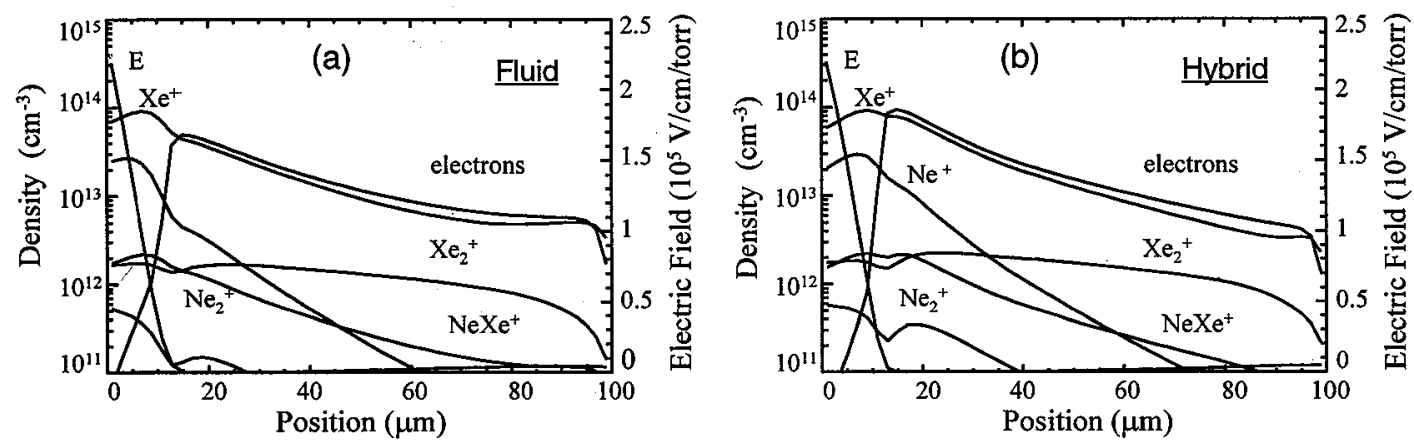

Figure 6: Spatial distributions of the charged particle densities and electric field at the time of peak current from (a), the fluid, LFA model, and (b), from the hybrid fluid - Monte Carlo model

It is also interesting to note that the increase of xenon excitation in the plasma, on the anode side, which had been observed and discussed in Meunier et al. [4] (and attributed to a transient effect at the instant of peak current) is also predicted by the hybrid model. The profile and intensity of excitation in the plasma predicted by the hybrid and fluid models are however not identical.

Figure 6 shows the spatial profiles of the charged particle densities in the same conditions as Fig. 5, at the instant of peak current. The comparison is quite satisfactory although some differences can be observed between the predictions of the fluid and hybrid models: as expected, the ion density in the sheath is larger in the results from the fluid models than in those of the hybrid model, and the reverse is true for the plasma density.

Figure 7 shows the mid-margin and current half-width as a function of xenon concentration, deduced from the fluid and hybrid models. It appears from Fig. $7 \mathrm{a}$ that the mid-margin voltage predicted by the 
fluid model is $15-20 \mathrm{~V}$ below the mid-margin voltage deduced from the hybrid model. However the trends predicted by both models are in good agreement. The current half-width is about 3 ns longer in the hybrid model. The current pulse is shorter in the fluid case because the ions are created closer to the dielectric layer; they are created deeper in the plasma, due to non local effects, in the hybrid model.
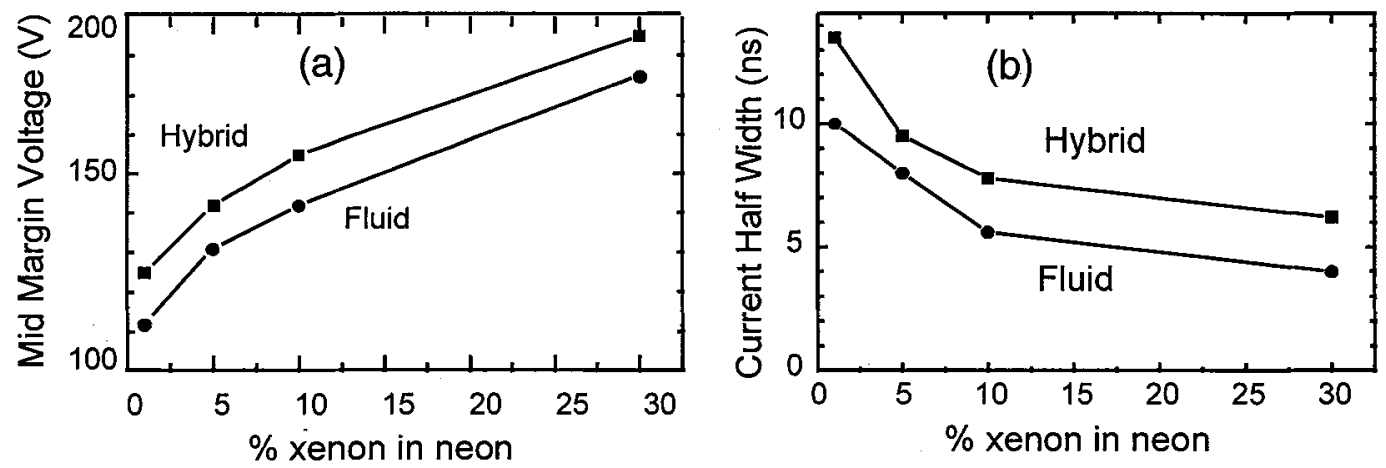

Figure 7: Comparisons of (a) the mid-margin voltage and (b), the current half width (at mid-margin) as a function Xe concentration, calculated with the fluid LFA model, and with the hybrid model

The current pulse and voltage across the gas gap are displayed in Fig. 8a. They are very similar to the results predicted by the fluid model (see Meunier et al. [4] ). The times to breakdown predicted by both models may however be quite different if the ionization coefficients used in the fluid models are not identical to those which would be calculated under uniform field conditions with the Monte Carlo simulation (this is the case in the calculations above since the ionization coefficients in the fluid model have been obtained from solutions of a Boltzmann equation corresponding to a Pulsed Townsend swarm experiment and not to a Steady State Townsend experiment).

Note finally that the UV efficiency of the discharge calculated with the fluid and hybrid models is practically identical (Fig. $8 \mathrm{~b}$ ).
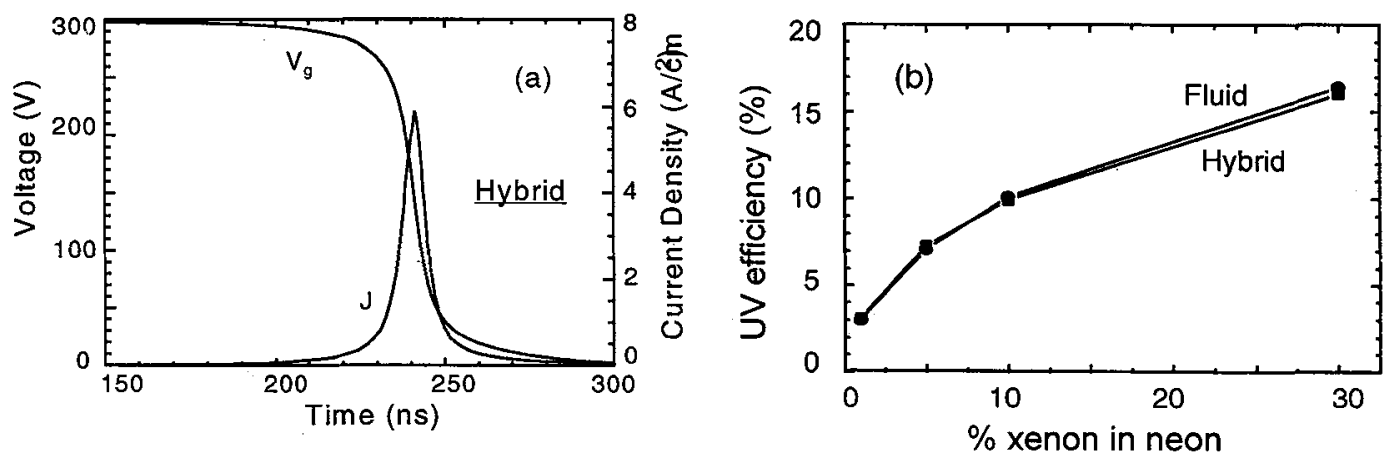

Figure 8: (a) Time evolution of the current and gas gap voltage obtained with the hybrid model (conditions of Fig. 5); (b) comparisons of the UV discharge efficiency calculated at mid-margin with the hybrid and fluid models for different xenonneon mixtures.

\subsection{Sensitivity to uncertainties in data}

The approximations made in the transport equations are a source of error as discussed in the previous section. Another source of error which is often more difficult to quantify is related to the uncertainties in 
the basic data which are used in the models. In our problem these data are the electron neutral crosssections (used in the hybrid model, or to calculate the transport coefficients), the transport coefficients (ionization coefficients, mobility ...), and the secondary electron emission coefficients. The most important unknown in a PDP discharge model is undoubtedly related to the secondary emission coefficient. We have only a rough estimation of the secondary emission due to neon ions on $\mathrm{MgO}$ $\left(\gamma_{\mathrm{Ne}} \sim 0.3-0.5\right)$ but we do not know at all how this coefficient depends on ion energy in the energy range of interest. The secondary emission coefficient for xenon ions on $\mathrm{MgO}$ is not known although it seems reasonable to assume that it is at least 10 times smaller than $\gamma_{\mathrm{Ne}}$. The ionization coefficients and other charged particle transport coefficients are known with a better accuracy. The accuracy of these coefficients is, roughly speaking, not better than $\pm 10 \%$ even in the relative simple case of rare gases.
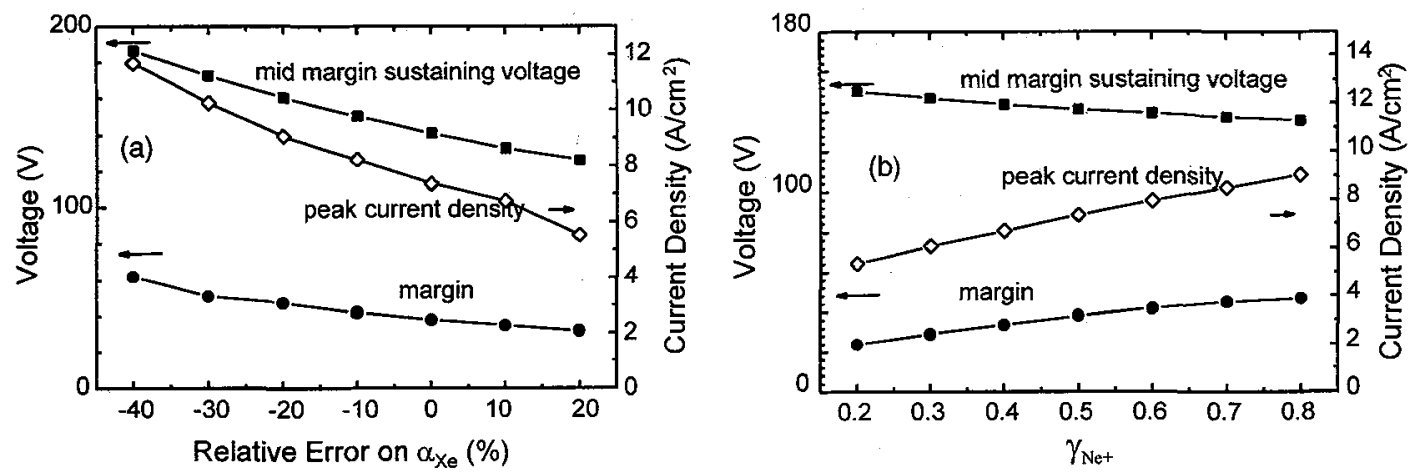

Figure 9: Sensitivity of the results (from the fluid LFA model) to the uncertainties in (a) xenon ionization coefficient, and (b), secondary electron emission by impact of neon ions on $\mathrm{MgO}$

In order to estimate the errors in the results which are due to uncertainties in the data, we have performed simulations where one of these parameters (ionization coefficient, secondary emission coefficient ...) was varied by a given percentage. Figure 9 shows the results of this study in the case of the xenon ionization coefficient (Fig. 9a) and the neon secondary electron emission coefficient (Fig. 9b). We see on this figure that the margin changes by a factor of 2 when the secondary emission coefficient of neon is changed between 0.2 and 0.5 . The mid-margin voltage decreases typically by $20 \mathrm{~V}$ when the xenon ionization coefficient is increased by $10 \%$.

\subsection{Are the fluid models accurate enough ?}

The conclusion of this section is that although the fluid LFA model does not capture all the complexity of the non local electron transport in the conditions of a plasma display panel cell, it can predict correctly the qualitative behavior and the trends. On a more quantitative point of view, the inaccuracies introduced by the fluid LFA model seem to be not larger than the inaccuracies introduced by the uncertainties in the basic data. Finally we mention that the computation time of the fluid LFA model is about 20-50 times shorter than the computation time needed by the hybrid model. This is an essential point for practical applications.

We believe that an efficient way to use discharge simulations in the optimization of plasma display panels is to use simple fluid models. These should be validated, for a few operating points, by comparisons with simulations based on hybrid or particle models. 


\section{DISCHARGE AND PLASMA FORMATION IN MATRIX AND COPLANAR GEOMETRY}

Figures 10 and 11 show typical results from a $2 \mathrm{D}$ fluid model of the discharge for matrix and.coplanar structures, respectively.

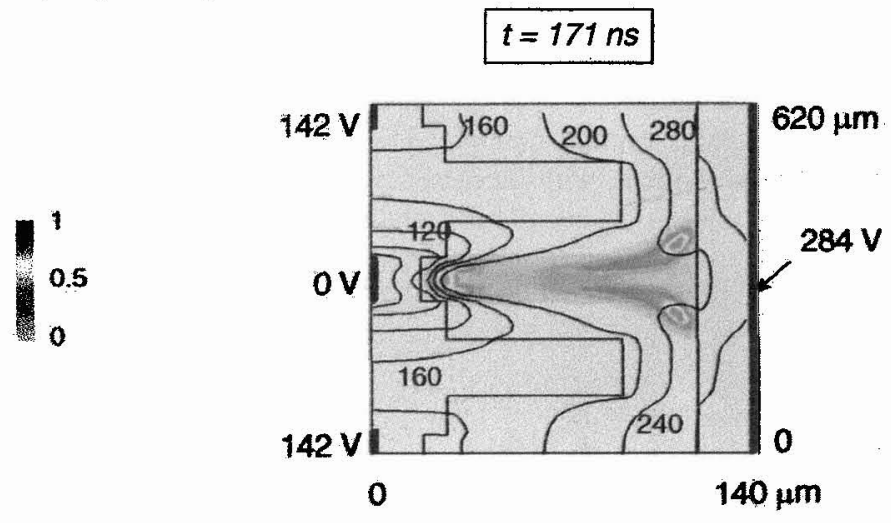

Figure 10: Equipotential curves and power dissipated in xenon excitation (unit $7.410^{4} \mathrm{~W} . \mathrm{cm}^{-3}$ ) at the instant of maximum current of a discharge in a matrix PDP (Xe-Ne 10\%-90\%, 560 torr). No memory charges are present before the discharge pulsc.

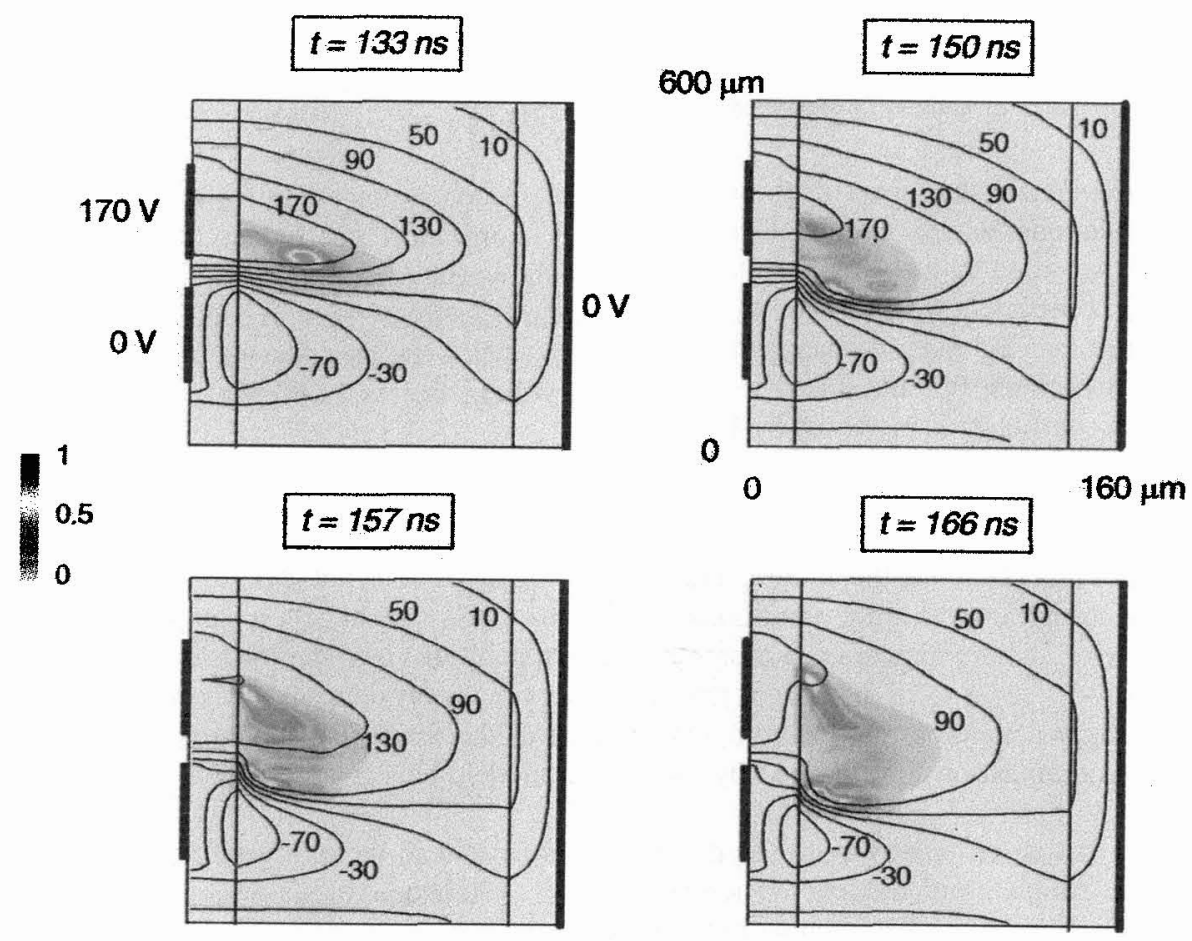

Figure 11: Equipotential curves and power dissipated in xenon excitation at four different times of a discharge pulse in a coplanar PDP cell (Xe-Ne 10\%-90\%, 500 torr). Memory charges are present before the discharge pulse and the discharge operates in the ON state of the sustaining regime. The units of the color scale for the power dissipated into xenon excitation are $1.8,5.5,7.9,3.810^{4} \mathrm{~W} . \mathrm{cm}^{-3}$, for $\mathrm{t}=133,150,157$, and $166 \mathrm{~ns}$ respectively. 
In the simulation of Fig. 10 the discharge was supposed to be in the OFF state before the voltage pulse, and no memory charges were present on the dielectric surfaces before the pulse. One cell and two half-cells are included in the simulation domain. Symmetric boundary conditions are assumed on the top and bottom of the simulation domain. We see in Fig. 10 the intense xenon excitation in the cathode region next to the left electrode. The equipotenial curves show the high field sheath next to the dielectric surface on the left side. Xenon excitation also occurs next to the dielectric surface on the anode side. This is related (see, e.g. [6], [9]) to the charging of the dielectric layers by electrons. The charging of the surface induces a local drop of the potential. The electrons which continue to flow to the surface move to the higher potential region along the dielectric surface. The electric field associated with the potential gradient along the surface appears clearly in Fig. 10 (see the shape of the potential line at $240 \mathrm{~V}$, next to the right dielectric surface) and is large enough to accelerate the electrons and induce significant xenon excitation.

The simulation of Fig. 11 corresponds to a discharge pulse in the sustaining regime of a coplanar cell. Periodic boundary conditions are assumed on the top and bottom of the simulation domain. Memory charges have been deposited by the previous pulse and are present on the dielectric surfaces at the beginning of the pulse of Fig. 11. The equipotential curves at the beginning of the current pulse ( $t=133 \mathrm{~ns})$ show the effect of the presence of the memory charges on the dielectric surface. The potential on the dielectric surface above the cathode (left electrode at 0 potential) is below $-70 \mathrm{~V}$ and the potential on the dielectric surface above the anode (left electrode at $170 \mathrm{~V}$ ) is larger than $170 \mathrm{~V}$. The applied voltage (sustain voltage, $170 \mathrm{~V}$ ) between the coplanar electrodes is smaller than the breakdown voltage in these conditions. The combination of the potential drop due to the memory charges and the voltage across the electrodes lead to a total voltage drop larger than the breakdown voltage in the gas gap above the coplanar electrode. A discharge is therefore initiated. At $t=133 \mathrm{~ns}$, the plasma has already formed above the anode. Xenon excitation is maximum above the anode, at the boundary between the large field region between the coplanar electrodes and the lower field region above the anode. At $t=150 \mathrm{~ns}$ the plasma has expanded, as shown by the extension of the low field region above the anode. The ion sheath tends to bend the equipotential curves above the cathode and the position of maximum excitation moves with the high field region. This motion continues at time $t=157 \mathrm{~ns}$ and $\mathrm{t}=166 \mathrm{~ns}$. The potential along the dielectric surface decreases above the anode because of the charging by electrons and increases slowly above the cathode due to the charging by ions. It is interesting to note that significant xenon excitation also occurs above the anode after $t=150 \mathrm{~ns}$. This increase in xenon excitation above the anode is due to the same phenomena as in the matrix case (Fig. 10). The potential quickly drops above the anode due to electron charging of the dielectrics and the resulting electric field parallel to the surface (see the first potential line above the anode at $\mathrm{t}=166 \mathrm{~ns}$ ) is large enough to accelerate the electrons and induce xenon excitation.

Simultaneous emission of the plasma above the cathode and anode of a coplanar plasma display cell has been observed by the group of Prof. Uchiike at Hiroshima University [17]. These authors used an ultra-high-speed electronic camera to measure the space and time evolution of the infrared emission of xenon. We believe that the observed emission above the anode in the experiments of Zhang et al. [17] is due to the electric field induced by the charging of the dielectric, as described above. Further work is however needed to confirm this point.

\section{CONCLUSION}

Physical models of the discharge occurring in plasma display panels are now being used in research laboratories as well as in industry. They provide very useful guidance in the design of the discharge cell and in the choice of operating conditions. They may, in the near future, play a key role in the search for better and more efficient plasma display panels.

Very few results from plasma diagnostic experiments have been published in the conditions of plasma displays, and there is a lack of reliable, published experimental data even on relatively simple questions such as the breakdown curves in a coplanar geometry. Since space and time resolved spectroscopic measurements are difficult in the conditions of a plasma display cell, we believe that 
experiments on larger cells and for lower pressure could be very useful to validate the models, to guide the optimization of the cell geometry, and to aid in the research of more efficient structures.

Although acceptable for practical use in some applications, several characteristics of PDPs need to be improved in order to achieve better accepted products and increase the market share [1][2]:

- luminous efficacy, which is about $1 \mathrm{~lm} / \mathrm{W}$ today, and must be increased up to a goal of $2 \mathrm{~lm} / \mathrm{W}$.

- life time, about 10000 hours now, must be more than 20000 hours in the mid term.

- image quality, related to addressing speed and priming issues, is not yet equivalent to that of Cathode Ray Tubes.

In order to move forward in the above characteristics, engineers need a better understanding of the physics underlying PDPs:

- an accurate energy budget of the complete system including discharge, phosphors, and cell geometry [18] shares is needed.

- a better knowledge about surface mechanisms: secondary emission and sputtering on $\mathrm{MgO}$

- a better view on addressing mechanisms and limitations: minimum time between two writing or erase operations, priming problems at low duty cycles.

The effort and results presented in this paper contribute to some of these items.

\section{Acknowledgments}

This work is partially supported by Thomson Tubes Electroniques.

\section{References}

[1] Deschamps J. and Doyeux H., Physics World June 1997 p. 39

[2] Deschamps J. and Doyeux H., "Color PDP development: an exciting and ever progressing story", International Display Workshop, IDW'96 (1996) p. 275

[3] Shinoda T., Wakitani M., Nanto T., Kurai T., Awaji N., and Suzuki M., "Improvement of Luminance and Luminous Efficiency of Surface Discharge Color AC PDP", Proceedings of the Society for Information Display Symposium, SID 91 Digest (1991) p. 724

[4] Meunier J., Belenguer P., and Boeuf J. P., J. Appl. Phys. 78 (1995) 731

[5] Boeuf J. P. and Doyeux H., Europhysics News 27 (1996) 46

[6] Boeuf J. P. and Pitchford L. C., IEEE Trans. Plasma Sci. 24 (1996) 95

[7] Campbell R. B., Veerasingam R., and McGrath R. T., IEEE Trans. Plasma Sci. 23 (1995) 698

[8] Veerasingam R., Campbell R. B., and McGrath R. T., IEEE Tran. Plasma Sci. 24 (1996) 1411

[9] Punset C., Boeuf J.-P., and Pitchford L. C., J. Appl. Phys. (1997) (submitted)

[10] Shinoda T., "The AC plasma display panel", International Display Workshop, IDW'95 (1995) p. 29

[11] Takayama O., "Innovation and commercial viability of large area plasma display through Fujitsu's continued R\&D activities", International Display Workshop, IDW'96 (1996) p. 7

[12] Sato S., Yamamoto H., Shirouchi Y., Iemori T., Nakayama N., and Morita I., IEEE Trans. Electron Devices 23 (1976) 328

[13] Makino M., Yoshioka T., Ueoka M., and Saito T., "Non-uniform electric field influence on firing voltage of surface discharge AC-PDPs", International Display Workshop, IDW'96 (1996) p. 259

[14] Choi K. C. and Whang K. W., IEEE Trans. Plasma Sci. 23 (1995) 399

[15] Boeuf J. P. and Pitchford L. C., IEEE Trans. Plasma Sci. 19 (1991) 286

[16] Fiala A., Pitchford L. C., and Boeuf J. P., Physical Review E 49 (1994) 5607

[17] Zhang S., Uchiike H., Harano Y., and Yoshoda K., "Consideration to improve luminance of AC plasma displays based on the discharge characteristics by using an electronic camera", Proceedings of the Society for Information Display Symposium, SID 97 Digest (1997) p. 225

[18] Doyeux H.,"Luminance and luminous efficacy of PDPs", International Display Workshop, IDW'95 (1995) p. 53 